\title{
Primary extranodal non-Hodgkin lymphomas of the uterus and the breast: report of three cases
}

\author{
Mario Adelfio Latteri, Calogero Cipolla, Vittorio Gebbia*, Gabriella Lampasona, Carmela Amato and \\ Nicolò Gebbia*
}

\author{
Department of Anatomy and Surgery, Division of Surgical Oncology, *Service of Chemotherapy, \\ Institute of Pharmacology, Policlinico, University of Palermo, and †Division of Obstetrics and Gynecology, \\ Hospital 'Villa Sofia', Palermo, Italy
}

\begin{abstract}
The authors describe one case of a rare primitive non-Hodgkin lymphoma of the uterus, and two cases of primary non-Hodgkin lymphoma of the breast. Histologically, the uterine lymphoma, although clinically confined to the uterus, was a diffuse large cell lymphoma, group $G$ according to the Working formulation for Clinical Usage. The two cases of breast lymphoma were a centrocytic-centroblastic and a lymphoplasmocytoid non-Hodgkin lymphoma, respectively. All cases were initally treated with radical surgery plus radiotherapy, but the first patient showed an early recurrence at distant sites, which required systemic cytotoxic chemotherapy. The patient with uterine nonHodgkin lymphoma received a very intense regimen--i.e. the ProMACE-Cytabom-because of the unfavourable histology, while the two patients with primary breast non-Hodgkin lymphoma received less aggressive CHOP and CVP chemotherapy. All patients are still alive and free of disease 3 to 6 years after initial diagnosis. These cases stress the systemic nature of non-Hodgkin lymphomas even if apparently localized to a single extranodal organ. Thus, although a definitive therapeutic strategy cannot be drawn from the rare and occasional reports in the medical literature, primary extranodal lymphomas require integrated multimodality therapy with radiotherapy and/or chemotherapy.
\end{abstract}

Key words: extranodal non-Hodgkin lymphoma; uterus; breast; surgery; chemotherapy.

\section{Introduction}

Non-Hodgkin lymphomas represent approximately $3 \%$ of all malignances in the United States which account for about 13,000 deaths/year. ${ }^{1,2}$ Although in the medical literature data concerning primary extranodal non-Hodgkin lymphomas (NHL) are rare and not unifocal, the diagnosis of a primary extranodal NHL is a relatively frequent occurrence in clinical oncology. In fact, up to one third of NHL may have an extranodal origin, expecially in the gastrointestinal and the naso-oropharyngeal regions. However, extranodal NHL may originate not only in organs containing well recognized lymphoid tissue such as small bowel Peyer's patches or tonsils, but also in individual organs that usually do not contain lymphoid tissue. ${ }^{3,4}$ In this paper we report one case of a primary NHL of the uterus and two cases of primary NHL of the breast. Both localizations, in particular the uterine one, are extremely rare forms of extranodal NHL. In fact, in a large review of 1467 extranodal NHL collected over 14 years at the National Cancer Institute of Bethesda, Maryland, USA, primary NHL of the uterus and the breast represented $0.002 \%$ and the $2.2 \%$ respectively, of the whole series. ${ }^{4}$ Moreover, NHL represent only $0 . \mathrm{I}-0.5 \%$ of all breast tumours. ${ }^{4}$

\section{Case 1: Primary NHL of the corpus uteri}

In June 1992 a 46-year-old white female was admitted at the Division of Gynecology of an affiliated Hospital in the city of Palermo for an unexplained persistent uterine haemorrhage. History was negative for infections, neoplastic disorders, and immunodeficiency diseases. Physical examination failed to reveal any sign of disease, except for the uterine involvement. Gynaecological examination failed to detect significant abnormalities. No B symptoms were present, and all routine haematological and serum chemistry tests were normal except for a mild normocromic normocytic anaemia and a moderately increased ESR $(54 \mathrm{~mm} / \mathrm{h})$. A hysteroscopy showed an area of hypertrophic abnormal mucosa localized in the fundus uteri which was biopsied. Histological analysis showed the presence

*Ccorrespondence to: Mario Adelfio Latteri, Head Division of Surgical Oncology, via Marchese Ugo n. 56, Palermo, Italy. of a uterine neoplasm represented by undifferentiated large cells with a sarcomatous appearance, which prompted the pathologist to put forward a tentative diagnosis of uterine sarcoma. Thus, the patient underwent total laparohysterectomy with bilateral oophorectomy without any significant post-surgical complication. Definitive histological analysis of the specimen surprisingly revealed the presence of a diffuse large cell NHL, group G, according to the Working Formulation of NHL for Clinical Usage. ${ }^{\text {s The neoplastic }}$ infiltration was confined to the corpus uteri, while the ovaries were normal.

The patient was then referred to our Institution where she sought oncological advice at the end of August 1992. Extensive staging with physical examination, chest X-ray, abdominal sonogram, ORL examination, bilateral bone marrow biopsy, total body CT scan, abdomino-pelvic NMR, and routine haematological and chemistry tests, failed to detect any sign of disease. Tumour markers, including beta 2-microglobulin, were negative. Thus, a diagnosis of primary uterine NHL was eventually carried out.

The patient was informed of the rarity of her disease, and was rapidly submitted for radiotherapy to the surgical field and locoregional lymph node areas. Radiotherapy was given with an inverted $Y$ field for a final dose of 45 gray ( 2 Gy/day $\times 5 /$ week) from September to the end of November 1992. Mild nausea was the only complaint during radiotherapy. Soon after completion of radiotherapy, clinical examination revealed the presence of four mobile, painless, small $(1-2 \mathrm{~cm})$ lymph nodes in the anterior area of the left axilla, one of which was surgically removed. A total body CT scan did not show any other enlarged or suspect lymph nodes elsewhere. Pathological analysis again discovered the presence of a NHL with the same characteristics as the uterine one. Thus, the patient was given eight fullecycles of chemotherapy according to the ProMACEMOPP regimen. ${ }^{6}$ Recombinant granulocyte colony stimulating factor $5 \mu \mathrm{g} / \mathrm{kg} /$ day was employed subcutaneously as necessary to prevent severe leukopenia enabling planned dose-intensity. During chemotherapy the patient complained of mild mucositis and complete transient alopecia, but vomiting was virtually absent due to the use of i.v. ondansetron. After completion of chemotherapy the patient was clinically and radiologically restaged also with total body CT scan without evidence of disease. Thus a complete response according to the WHO criteria was obtained. ${ }^{7}$ The patient is still alive and free of disease, 30 months from surgery 
Primary NHL of the breast

\section{Case no. 1}

In September 1987 a 47-year-old white female was admitted to our Institution for mastalgia. History was negative for infectious, neoplastic, lymphoproliferative, and immunodeficiency disease. Physical examination revealed the presence of a small $(\mathrm{lcm})$ painless mass in the upper outer quadrant of the right breast with a negative axilla. The breast lesion was smooth, mobile, and well defined. No suspicious lymphnodes were found elsewhere. Clinically, liver and spleen were not enlarged. No B symptoms were present. The results of routine laboratory tests revealed an increased ESR $(62 \mathrm{~mm} / \mathrm{h})$, a mild hypocromic anaemia $(\mathrm{Hb} 11.2 \mathrm{~g} \%$ ), and a relatively low serum iron $(40 \mu \mathrm{g} \%)$. Breast sonogram confirmed the presence of the solid nodule surrounded by a small group of calcifications, but aspiration of the mass was not diagnostic. An open biopsy was then performed the nodule completely removed, and histological analysis discovered the presence of a malignant centrocytic-centroblastic NHL according to the Kiel classification." The margins of the surgical specimen were histologically free of tumour.

The patients was then thoroughly staged with chest X-ray, abdominal sonogram, $99 \mathrm{Te}$ bone scan, CT scan of the head/neck, thorax and the abdomen, and bilateral bone marrow biopsy with a Jamshidi needle. Routine haematological tests and serum chemistry were normal. All the above-mentioned examinations failed to discover any sign of lymphomatous infiltration, and the disease was thus considered a primary NHL of the breast, stage IE according to the Ann Arbor staging system. ${ }^{5}$

The patient was subsequently given radiotherapy with a mantle field for a total of 45 gray ( $2 \mathrm{~Gy} / \mathrm{day} \times 5 /$ week), and then closely followed-up. Forty-six months after surgery a nodal recurrence in the right axilla, inside the previous radiotherapy field, was found. The patient then underwent systemic chemotherapy with cyclophosphamide, vincristine, adriamycin and prednisone (CHOP regimen). The major side-effects were alopecia and granulocytopenia, but both were transient. After a total of eight cycles the patient was extensively restaged and a complete response according to the WHO criteria was found. ${ }^{7}$ The patient is still alive without signs of disease after 6 years from diagnosis.

\section{Case no. 2}

In January 1990 a 54-year-old white female was seen at our breast screening outpatient clinic for a painless mass in the lower outer quadrant of the left breast. Medical history was negative for neoplastic disorders and, in particular, for lymphoproliferative diseases. Clinical examination discovered a $3 \times 3.5 \mathrm{~cm}$ smooth and mobile mass in the left breast. Non-palpable lymph nodes were found. Mammography showed a nearly round opacity with well-defined margins and without calcifications, which was aspirated for cytological analysis. Cytologic analysis revealed the presence of rare epithelial cells with hyperchromic and hypertrophic nuclei mixed with several lymphocytes. An open surgical biopsy of the lesion was then performed, and definitive histological analysis revealed the presence of a lymphoplasmocytoid non-Hodgkin lymphoma ( $\mathrm{k}$ chain) according to the Kiel classification ${ }^{5}$. Thus, no further surgery was carried out, and the patient was staged as described above for Case 1. The disease was considered a primary NHL of the breast stage $1 \mathrm{E}$.

The patient was then given mantle field radiotherapy for a total final dose of 47 gray, plus systemic chemotherapy with the CVP regimen (cyclophosphamide, vincristine and prednisone) for eight cycles. Adriamycin was not included in the chemotherapy regimen due to the lymphoplasmocytoid type of NHL which is considered a low malignant lymphoma. After completion of radiotherapy and systemic chemotherapy, restaging did not reveal any sign of disease. To date the patient is still alive in good clinical conditions without any sign or symptom of disease after a follow up of 46 months.

\section{Discussion}

Organs containing well-recognized areas of lymphoid tissue, such as the stomach, tonsils, small bowel and skin, are by far the most common sites of origin of primary extranodal non-hodgkin lymphomas ${ }^{3.4}$. However, a primary extranodal NHL may occasionally occur in many other tissues which usually do not contain well identifiable lymphoid areas ${ }^{4}$

Primary extranodal uterine NHL is an extremely rare form of malignant lymphoproliferative neoplastic disease of the uterus. In the absence of distant nodal metastases no particular symptoms are characteristic of such disease, and clinically it is not distinguishable from other more common malignancies of the uterus. In the case reported above, surgery was performed due to the initial diagnosis of uterine sarcoma, but in view of the lymphomatous nature of the disease surgery plays more of a diagnostic role than a therapeutic one even if removal of the bulky uterine neoplasm retains its clinical importance for debulking. In fact, primary extranodal NHL, even if localized at diagnosis, tends to behave as a systemic disease as confirmed by the reported patient who showed early distant recurrence after radical surgery and radiotherapy.

Primary extranodal NHL of the breast is also a very rare neoplastic disorder, representing $0.5 \%$ of all neoplastic diseases of the breast, and only $2.2 \%$ of all forms of primary extranodal $\mathrm{NHL}^{9-11}$. Its clinical recognition is particularly difficult as there are no clinical or radiological signs peculiar to this disease. The mean age of patients with breast NHL varies from 45 to 65 years in different series, but generally the incidence seems to increase with age. This age range is not characteristic of NHL since it overlaps with the peak of incidence of breast carcinoma. However, other authors have reported the age pattern of incidence as a bimodal distribution: a younger group of patients with age mode of 33 years and a second older group with an age mode of 63 years ${ }^{11}$. Few authors reported a prevalence of primary NHL in the right breast. The predominance is statistically significant compared to the left breast in which breast carcinoma is more frequent. Although some authors reported that histopathological type and clinical staging play a significant prognostic role ${ }^{10.13}$, other authors failed to find such an association ${ }^{11}$. The role of surgery in primary breast NHL is mainly diagnostic since the lymphoproliferative disorder is a widespread disease often requiring systemic therapy. In fact, even if local control could be effectively achieved with radiotherapy alone, distant spread of the disease remains a major clinical problem since most patients will sooner or later develop distant recurrence despite optimal locoregional therapy ${ }^{12,13}$. However, Abbes et al concluded that exclusive radiation therapy could be justified in low-malignacy $\mathrm{NHL}$, and systemic chemotherapy represented the mainstay of therapy for intermediate- and high-malignacy forms ${ }^{13}$. In this paper we report two cases of primary breast NHL: the first patient had an intermediate malignancy NHL, showed an early relapse after locoregional treatment and thus received systemic chemotherapy which was successful in controlling the disease; the second patient had a low grade malignancy NHL, was treated with non-aggressive systemic chemotherapy before any relapse appeared. Survival of both cases is in the range reported by other authors. ${ }^{9.10 .13}$

In conclusion, primary extranodal NHL of the uterus and the breast are very rare neoplastic disorders, for which diagnosis is only obtained after a definitive histological and immunocytochemical analysis. The role of surgery, beside removing the primary mass, is mainly diagnostic since in most cases an extranodal NHL should be considered as a systemic disorder even when the disease seems well localized. Although a definitive therapeutic strategy cannot be drawn from the occasional reports in the medical literature, of extranodal NHL arising in non-lymphoid organs, primary extranodal NHL, expecially intermediate- and high-malignancy forms, in our opinion, require integrated multimodality therapy with adjuvant radiotherapy and systemic chemotherapy, particularly, in those cases with poor prognostic factors.

\section{References}

1. Thomas DB. Cancer epidemiology and prevention. In: Moossa AR, Robson MC, Schimpff SC (eds), Comprehensive Textbook of Oncology. Baltimore: Williams \& Wilkins, 1986: 3-27.

2. Little C, Longo DL. Non-Hodgkin lymphomas: epidemiology, etiology, pathology, natural history, and disease evolution. In: Moossa AR, Robson MC, Schimpff SC (eds). Comprehensive 
Textbook of Oncology. Baltimore: Williams \& Wilkins, 1986: 574-92.

3. Banfi A, Bonadonna G, Carnevali G, Oldini C, Salvini E. Preferential sites of involvement and spread in malignant lymphomas. Eur J Cancer 1968; 4: 319-24.

4. Freeman C, Berg JW, Cutler SJ. Occurrence and prognosis of extranodal lymphomas. Cancer 1972; 29: 252-60.

5. De Vita VT, Helmann S. Hodgkin disease and the non Hodgkin's lymphomas. In: De Vita VT, Hellman S, Rosenberg SA (eds), Cancer: Principles and Practice of Oncology. JB Lippincott Co., 1982: 1331-401.

6. Miller TP, Dahlberg S, Jones SE. ProMACE-CytaBOM is active with acceptable toxicity in patients with unfavorable nonHodgkin lymphoma: a Southwest Oncology Group study. Proc Am Soc Clin Oncol 1987; 6: 197.

7. Miller AB, Hoogstraten B, Staquet M, Winkler A. Reporting results of cancer treatment. Cancer 1981; 47: 207-14.

8. Smith MR, Brustein S, Strauss D. Localized non-Hodgkin lymphoma of the breast. Cancer 1987; 59: 351-4.
9. Brustein S, Kimmel M, Lieberman PH, Filippa DA, Rosen PP. Malignant lymphoma of the breast. Ann Surg 1987; 205: 144 50.

10. Jeon HJ, Akagi T, Hoshida Y, Hayashi K, Yoshino T, Tanaka T, Ito J, Kamei T, Kawabata K. Primary non-Hodgkin malignant lymphoma of the breast. Cancer 1992; 70: 2451-9.

11. Chen MG, Prosnitz LR, Gonzalez-Serva A, Fisher DB. Results of radiotherapy in control of stage I and II non-Hodgkin lymphoma. Cancer 1979; 43: 1245-54.

12. Levitt SH, Bloomfield CD, Frizzera G, Lee CKK. Curative radiotherapy for localized diffuse histiocytic lymphoma. Cancer Trest Rep 1980; 64: 175-7.

13. Abbes M, Persch M, Bongain A, Schneider M, Thyss A, Hery M, Ettore R. Localization mammaire primitive des lymphomes malins non hodgkiniens. J Chir 1991; 128: 65-70.

Accepted for publication 22 April 1994 